\title{
The development of Chinese elderly people PIM list
}

\author{
Sai Wang ${ }^{1}$, Yan Yan ${ }^{2}$, Xiaolan Zhang ${ }^{3}$, Lan Zhang ${ }^{1}$, Yuqin Wang ${ }^{1}$ \\ ${ }^{1}$ Xuan Wu Hospital, Capital Medical University, China, ${ }^{2}$ Beijing Pharmaceutical centralized purchasing service center, \\ China, ${ }^{3}$ Beijing Jing Lu He Hospital, Capital Medical University, China
}

Background: Potentially inappropriate medication (PIM) is a kind of high-risk drug. Older people may bear more potential adverse effects than expected benefit of such drugs. Following the release of the BEERS Criteria by American geriatrics experts in 1991, many countries issued their own PIM criteria. However, the PIM criteria has not been established in China and the BEERS Criteria is widely used in clinical but not fully applicable. So it is urgent to develop PIM criteria that meets the needs of elderly patients in China.

Method: Chinese clinical and pharmacology experts used three rounds of Delphi method based on the PIM lists of the United States, Canada, Japan and other eight countries' PIM criteria, combined with the SADR data of China National Center for ADR Monitoring, ADR monitoring center in the People's Liberation Army, Beijing Center for ADR Monitoring and ADR data from Beijing 22 hospitals, established Chinese elderly people PIM list, which contained PIM use in aged and PIM in aged people under morbid state.

Result: Anti-infection-resistant drugs, neurologic drugs, cardiovascular system drugs and other 13 class, 72 species of drugs were selected in PIM use in aged, each medicine had 1-6 risk points. Combination with the monitoring characteristics of drug and ADR in elderly patients in China, clopidogrel, clindamycin, vancomycin and gatifloxacin were added. The list was divided according to the result of expert evaluation into 35 high risk medication and 37 low risk medication. In addition, according to the frequency of drug use, the medications were divided into A and B two categories, including 24 medication or medication classes as the preferred alert medications (A), 48 medication or medication classes as routinely alert medications (B). PIM in aged people under morbid state contained 74 points of druginduced risks of 44 species (categories) of drugs under 27 kinds of morbid states.

Conclusion: Chinese aged people PIM list have been developed preliminarily, which can be taken as reference to intervention and evaluation of China's elderly medication. 